\title{
DANDY WALKER SYNDROME- A 13 YEARS OLD GIRL WITH WALKING DIFFICULTIES
}

\author{
KANIZ FATEMA ANONNYA ${ }^{1}$, MD REZAUL EKRAM ${ }^{2}$, PRANAB KUMAR MALLIK ${ }^{3}$, MOHAMMAD RAFIQUL ISLAM ${ }^{4}$, \\ MD. ROBED AMIN ${ }^{5}$
}

\begin{abstract}
Dandy-Walker malformation (DWM) is a uncommon intracranial congenital abnormality that affects the cerebellum and some of its components; particularly cerebellar vermis, fourth ventricle and is characterized by an enlarged posterior fossa. Although there is an extensive list of signs attributed to DWM, final diagnosis is solely dependent on imaging techniques as there are no signs that are characteristic of DWM. This article reports a case with DWM who was diagnosed by magnetic resonance imaging.
\end{abstract}

Received: 13 July 2015

Accepted: 3 November 2015

\section{Introduction}

Dandy-Walker malformation is a rare congenital abnormality that affects the cerebellum and some of its components; particularly hypoplasia of cerebellar vermis, a cystic dilatation of fourth ventricle and is characterized by an enlarged posterior fossa. ${ }^{1}$

Dandy-Walker malformation was originally described in 1887 by Sutton and further characterized by Dandy and Black fan in 1914 followed by Tagart and Walker in 1942. Benda finally labeled this disease as dandy walker in $1954 .^{2}$

Dandy-Walker complex has several variants, DandyWalker malformation (DWM) encompasses cystic dilatation of the fourth ventricle, complete or partial agenesis of cerebella vermis and enlarged posterior fossa while Dandy-Walker variant (DWV) comprises cystic posterior mass with variable hypoplasia of the cerebella vermis and no enlargement of the posterior fossa. However, the third variant mega-cisterna magna comprises enlarged cistern magna with normal cerebellar vermis and fourth ventricle. ${ }^{3}$

Infants with DWM may present with early signs such as vomiting, sleepiness, irritability, convulsions, unsteadiness and lack of muscle coordination. ${ }^{4}$

The clinical manifestations include psychomotor and growth retardation, hypotonia, strabismus, myopia, a short neck, microcephaly, brachycephaly, hypertelorism, antimongoloid slant of palpebral fissures, globulus large nose, large mouth with down turned corners, poorly lobulated ears, high arch palate, cleft palate, small hands and feet, clinodactyly, and the brachymesophalangy of the little fingers. 5

Although it is said that clinical examination cannot replace any imaging modalities, DWM is such a condition that require imaging modalities to diagnose the disorder. Even though there are many signs, none of these are characteristic to diagnose individuals as DWM and diagnosis is solely based on imaging techniques. The present manuscript reports a case encountered in Dhaka Medical College Hospital which was revealed as Dandy-Walker malformation by MRI.

\section{Case Report}

A female patient of age 13 years was brought to the department of medicine with the complaints of gradually progressive unsteadiness \& weakness of left side of the body for 18 months. She is a student of class three $\&$ she discontinued her study due to tendency of frequent fall. She had a history of pustular lesion involving whole of the scalp 3 months back which subsided by taking medication from local doctors. No history of headache, fever, convulsion, irritability or drowsiness. Her milestone of development in childhood was normal. According to mother, her mental, social \& emotional development is also normal. . But no history of development of

1. Medical officer, Department of Medicine, Dhaka Medical College Hospital

2. Assistant Registrar, Department of Medicine, Dhaka Medical College Hospital

3. Registrar, Department of Medicine, Dhaka Medical College Hospital

4. Assistant Professor, Department of Medicine, Dhaka Medical College Hospital

5. Associate Professor, Department of Medicine, Dhaka Medical College Hospital

Correspondence : Dr. Md. Robed Amin, Associate Professor of Medicine, Department of Medicine, Dhaka Medical College, Bangladesh. e-mail: robedamin@yahoo.com Cell-01711725787

Bangladesh J Medicine 2016; 27 : 33-36 

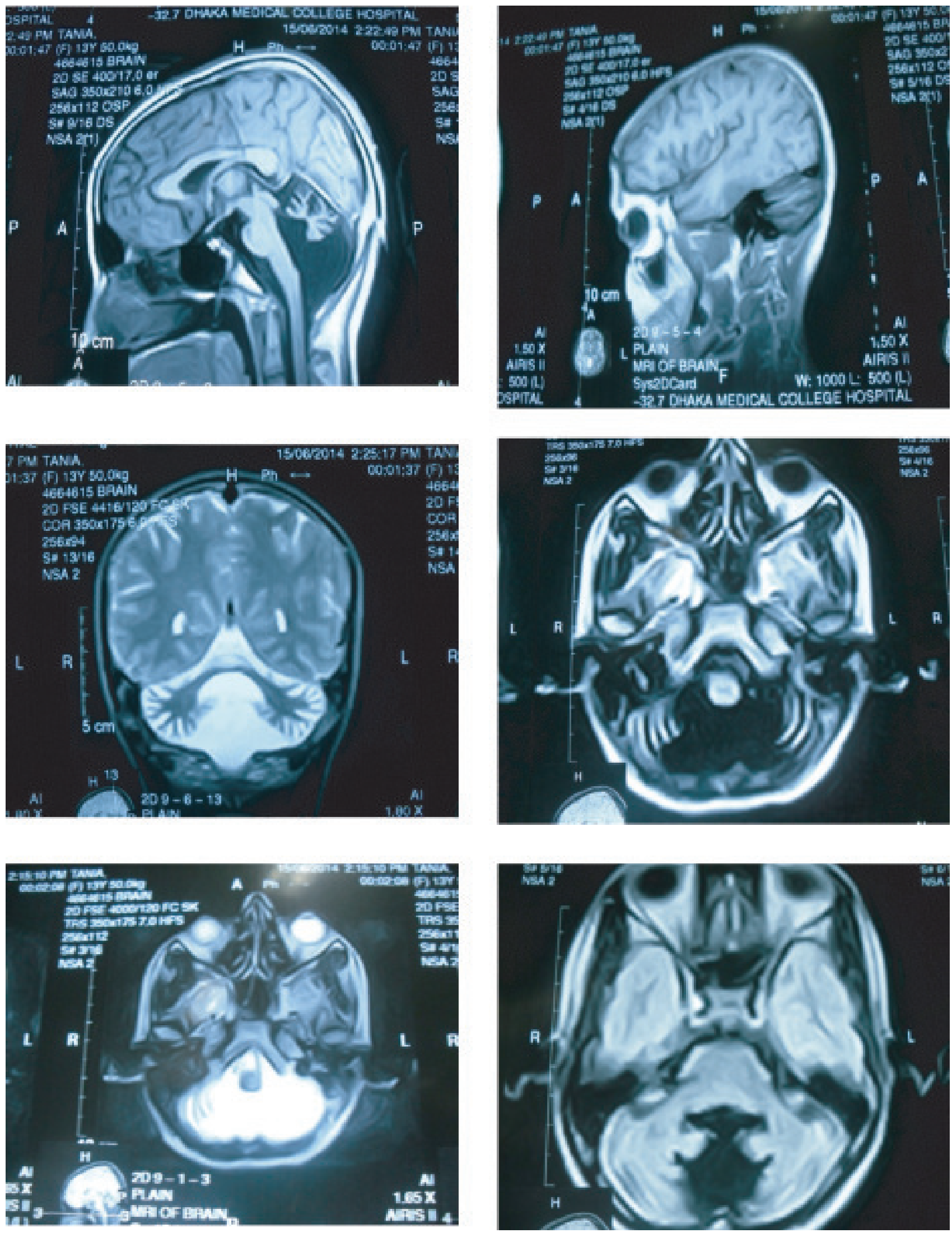

Fig.-1 
secondary sexual characteristics like menarche, thelarche, growth of pubic \& axillary hair. Her mother did not provide any history of medication during pregnancy. She was not diabetic or alcoholic. She was born at term and was the third child of nonconsanguineous parents and the only affected case in the family. Her mother did not give any history of abortion or still birth or child death.

She is co-operative, conscious, oriented. But her body built below average (age bor BMI chart showed she is at $5^{\text {th }}$ percentile according to BMI value-15 \& weight for age chart showed she is below $3^{\text {rd }}$ percentile- $27 \mathrm{~kg}$ ). Her GCS-15, Minimental State Examination revealed 30/30 .Stance \& gait apparently normal but tandem gait showed ataxia. Speech is normal (no dysarthria). Cranial nerves are intact including fundus. Muscle bulk below average in both upper \& lower limbs. Muscle power $4 / 5$ in both upper \& lower limbs.Superficial \& deep tendon reflexes are normal. No pendular jerks. All sensory examinations were normal. Finger finger nose test, dysdiadokokinesia is normal while heel shin test showed left sided dysmetria. Study of the MRI of Brain revealed a welldefined extra axial cystic lesion in the posterior fossa in the midline communicating with the fourth ventricle. There was an associated mild hypoplasia of the cerebellar vermis and folia, the posterior fossa was not enlarged, there is neither dilatation of occipital horns of lateral ventricles (colpocephaly) nor agenesis of corpus callosum. These findings thus paved to the diagnosis of Dandy-Walker variant.

\section{Discussion}

DWM occurs as an autosomal dominant inherited disorder and occur in one in 25000-35000 pregnancies. ${ }^{2}$ The syndrome can appear dramatically or develop unnoticed. The gene locus for DWM is $3 q 24^{6}$ and the presence of multiple congenital defects associated with DWM may shorten life span. ${ }^{6}$

Cases of Dandy-Walker malformation were reported more in females. ${ }^{7}$ Various predisposing factors were reported such as infections, cranial trauma, chronic disturbance in cerebrospinal fluid pressure, persistence of embryonic tissue, vascular lesions, teratogens, rubella, alcohol and maternal diabetes. ${ }^{2}$ Previous literature suggests that $40 \%$ individuals with DWM were normal intellectual while $40 \%$ had mental retardation and $20 \%$ were borderline. ${ }^{2}$

Cerebellum is believed to play a role in motor control, motor learning, and even cognition such as development of language and other cognitive skills. ${ }^{8}$ Thus, the effect of Dandy-Walker syndrome on intellectual development is variable, with some children having normal cognition and others never achieving normal intellectual development.

Paladini and Volpe ${ }^{9}$ in 2006 demonstrated that the degree of vermian hypoplasia correlates significantly with the occurrence and severity of mental retardation. Thus, it seems that the more abnormal the vermis is, the more poor the prognosis will be and our case had only mild hypoplasia of cerebellar vermis thus showing milder presentation.

Syndromes associated with DWM include "PHACE syndrome" (posterior fossa brain malformations, hemangiomas, arterial anomalies, coarctation of aorta and cardiac defects and eye abnormalities) 10 and Ellis-van Creveld syndrome. ${ }^{11}$ A large number of associated problems may also be present, such as hydrocephalus (often develops postnatally), atresia of the foramen of Magendie and atresia of the foramen of Luschka.

Through the use of modern diagnostic tools (e.g., ultrasound, CT, MRI, etc.), DWM is typically diagnosed in individuals before one year of age in approximately 76 to $80 \%$ of the cases. Historically, DWM was only found incidentally or by autopsy ${ }^{12}$.DWM are still a challenge in prenatal diagnosis. Technical developments in imaging, such as in threedimensional sonography and magnetic resonance, allow higher resolution and multiplanar images for an easier diagnose. There is a high rate of false positive, particularly before the 18 th week of gestation. It is advisable not to establish a final diagnose before that week. Adult cases of DWM for instance are usually diagnosed incidentally by neuroimaging methods or after minor head trauma as diagnosed in the present case ${ }^{13}$.

\section{Conclusions}

Dandy-Walker malformation is a condition that can be diagnosed by advanced imaging modalities which are expensive to use in developing countries. Extensive research is required to identify or diagnose the disease at the earliest.

\section{References}

1. Ecker JL, Shipp TD, Bromley B, Benacerraf B. The sonographic diagnosis of Dandy-Walker and DandyWalker variant: Associated findings and outcomes. Prenat Diagn. 2000; 20: 328-32.

2. Cardoso J, Lange MC, Lorenzoni PJ, Scola RH, Werneck LC. Dandy-Walker syndrome in adult mimicking myasthenia gravis. Arq Neuropsiquiatr. 2007; 65: 173-5.

3. Alam A, Chander BN, Bhatia M. Dandy walker variant: Prenatal diagnosis by ultrasonography. Med J Armed Forces India. 2004;60:287-9. 
4. Simpkins CJ. Ventriculoperitoneal shunt infections in patients with hydrocephalus. Pediatr Nurs. 2005;31:457-62.

5. Baburao V, Kerketta L, Seema K, Ghosh K. Dandy Walker malformations in the case of Partial Trisomy $9 \mathrm{p}(\mathrm{p} 12.1$ '! pter) due to maternal translocation $(9 ; 12)$ (p12.1; p13.3) Indian J Hum Genet. 2007;13:33-5.

6. National Institute of Neurological Disorders and Stroke. (2005, February) Dandy Walker syndrome information page. Available from: http:// www.ninds.gov/disorders/dandywalker. [retrieved on 2005 Jul 14].

7. Osenbach RK, Menezes AH. Diagnosis and management of the Dandy-Walker malformation: 30 years of experience. Pediatr Neurosurg. 1992; 18:179-89.

8. Ten Donkelaar HJ, Lammens M, Wesseling P, Thijssen HO, Renier WO. Development and developmental disorders of the human cerebellum. J Neurol. 2003;250:1025-36.

9. Paladini D, Volpe P. Posterior fossa and vermian morphometry in the characterization of fetal cerebellar abnormalities: A prospective threedimensional ultrasound study. Ultrasound Obstet Gynecol. 2006;27:482-9.

10. Lin $\mathrm{MC}$, Chen $\mathrm{CH}$, Chi CS. PHACE syndrome: Report of one case. Acta Paediatr Taiwan. 2003;44:37982

11. Jones KL. 5th ed. Elsevier Saunders; 1997. Smith's Recognisable patterns Human malformation.

12. Engelhard HH, Meyer JR. Adult-onset presentation of Dandy-Walker variant in siblings. Surg Neurol. 1995;44:43-7.

13. Sato K, Kubota T, Nakamura Y. Adult onset of the dandy-Walker syndrome. Br J Neurosurg. 1996; 10:109-12. 